\title{
Localization of Electronic States in Chain Models Based on Real DNA Sequence
}

\author{
Hiroaki Yamada ${ }^{1}$ \\ Aoyama 5-7-14-205, Niigata 950-2002, Japan
}

\begin{abstract}
We investigate the localization property of an electron in the disordered two- and three-chain systems (ladder model) with long-range correlation as a simple model for electronic property in a double strand of DNA. The chains are constructed by repetition of the sugar-phosphate sites, and the inter-chain hopping at the sugar sites come from nucleotide pairs, i.e., $A-T$ or $G-C$ pairs. It has been found that some DNA sequences have long-range correlation. In this paper we investigate the localization properties of the electronic states in some actual DNA sequences such as bacteriophages of escherichia coli, human chromosome 22 and histone protein. We will present some numerical results for the Lyapunov exponent (inverse localization length) of the wave function in the cases in comparison to the results for artificial sequence generated by an asymmetric modified Bernoulli map. It is shown that the correlation and asymmetry of the sequence affect on the localization in both the artificial and the real DNA sequences.
\end{abstract}

Key words: DNA, Sequence, Electronic states, Correlation, Localization, Delocalization, Lyapunov exponent

PACS: $42.50 . \mathrm{Hz}, 05.45 .+\mathrm{b}, 03.65 .-\mathrm{w}$

\section{Introduction}

The recent development of the nanoscale fabrication let us expect the utilization of the DNA wire as a molecular device $[1,2]$ and the realization of DNA computing [3]. Actually, the modern development enables us to measure the direct DNA transport phenomena [4,5]. Recently, Porth et al. measured the

$\overline{1}$ Corresponding author, electronic mail address: hyamada@uranus.dti.ne.jp, Former address: Faculty of Engineering, Niigata University, Ikarashi 2-Nocho 8050, Niigata 950-2181, Japan. 
nonequilibrium current-voltage $(I-V)$ characteristics in the poly $(\mathrm{G})$-poly $(\mathrm{C})$ DNA molecule attached to platinum lead at room temperature [4]. Cuniberti et al. explained the semiconducting behavior by considering the base-pair stack coupled to the sugar-phosphate (SP) backbone pair [6]. Iguchi also derived the semiconductivity and the band gap by using the ladder chain model of the double strand of DNA [7]. In above models, the existence of the SP backbone chains play an important role in the band structure due to the gap opening by the hybridization of the energy levels. Furthermore, recent ab-initio calculations in short segments show that the backbone chain of DNA might play an imprtant role for the entire electronic spectrum of the system $[8,9]$.

On the other hand, Tran et al. measured the conductivity along the lambda phage DNA ( $\lambda$-DNA) double helix at microwave frequencies using the lyophilized DNA in and also without a buffer [5]. The conductivity is strongly temperature dependent around room temperature with a crossover to a weakly temperature dependent conductivity at low temperatures. Yu and Song showed that the observed temperature dependent conductivity in the DNA can be consistently modeled, without invoking the additional ionic conduction mechanisms, by considering that electrons may use the variable range hopping for conduction and that electron localization is enhanced by strong thermal structural fluctuations in DNA [10]. Then the DNA double helix is viewed as a one-dimensional Anderson system. Carpena et al. and Roche used some real DNA sequence as the on-site energy in the tight-binding one-dimensional system to investigate the localizaton property of the wavefunctions $[11,12]$.

The transport property though DNA are still controversial mainly due to the tremendous difficulties in the setting up the proper experimental environment and the DNA molecule itself. Although many theoretical explanations for the charg transport phenomena have been suggested on the basis of the standard solid-state-physics approach such as polarons, solitons, hole hopping model on guanine sites $[13,14,15,16,17,18,19,20,21,22,23,24,25,1]$, the situation has been still far from unifying the theoretical scheme.

Moreover, as one of the realistic situation, it has been found that the base (nucleotide) sequence of the various genes has long-range correlation characterized by the power spectrum $S(f) \sim f^{-\alpha}(0.1<\alpha<0.8)$ in the low frequency limit $(f<<1)[26,27,28,29,30,31,32]$. As observed in the power spectrum, the mutual information analysis and the Zipf analysis of the DNA base sequence such as the human chromosome 22 (HCh-22), the long-range structural correlation exists in the total sequence as well as the short-range periodicity $[29,30,31,32]$. Eukaryote's DNA sequence has apparently periodic repetition in terms of the gene duplication. The correlation length in the base sequence of genes changes from the early eukayote to the late eukaryote by the evolutionary process. It seems that the long-range correlations tends to manifest in power spectra of the total sequences rather that in those of the exon part and the intron part 
separately [31].

The localization property of the single-chain disordered system with longrange correlation has been extensively studied [33,34,35]. Accordingly, to compare the localization nature of the electronic states in the real DNA sequence with that in the disordered sequence with long-range correlation is very interesting. In the present paper, we numerically give localization nature of the electronic states in some real DNA sequences such as bacteriopages of escherichia coli (E. coli), HCh-22 and histon H1. We also investigate the correlation effect on the localization property of the one-electronic states in the disordered chain models with a long-range structural correlation. We present some numerical results for the Lyapunov exponents of the wave function. In particular, it is found that the correlation of the sequence enhances the localization length and asymmetry of the distribution of the elements in the sequence affect on the localization.

Note that the real values for the biological molecule such as the ionization energy [36], the electronegativity and so on, are not used in the numerical calculation in the present paper. We used the simpler values as the parameters in order to show some basic localization and delocalization properties of the electronic states in the ladder models. We would like to mainly focus on (1)suggesting the model and (2)giving the preliminary numerical results of the electronic localization in the model with real DNA sequences.

Outline of the present paper is as follows. In the next section we introduce the simple model for DNA in order to investigate the electronic states. In the Sec. 3 we give the mapping rules of the real DNA sequences and an asymmetric modified Bernoulli map in order to generate artificially the correlated sequences. The numerical results for the Lyapunov exponent and the localization length in the systems are given in Sect. 4. The last section contains summary and discussion.

\section{Model}

We simplify and model the double strand of DNA by some assumptions. DNA double helix structure is constructed by the coupled two single strand of DNA. First, we ignore the twist of DNA as well as the complicated topology. In addition to the simplification, we consider only the $\pi$-electrons in the backbones and the base-pairs of the system. We also ignore the interaction between the electrons and restrict ourselves to the zero-temperature property.

Following the basic assumptions, consider the one-electron system described by the tightly binding model consisting of the two- or three- chains. The 
ladder model was first introduced by Iguchi [7] as a model for considering the electronic properties of a double strand of DNA. The Schrödinger equation is given as,

$$
\begin{aligned}
& A_{n+1, n} \phi_{n+1}^{A}+A_{n, n-1} \phi_{n-1}^{A}+A_{n, n} \phi_{n}^{A}+V_{n} \phi_{n}^{B}=E \phi_{n}^{A} \\
& B_{n+1, n} \phi_{n+1}^{B}+B_{n, n-1} \phi_{n-1}^{B}+B_{n, n} \phi_{n}^{B}+V_{n} \phi_{n}^{A}=E \phi_{n}^{B}
\end{aligned}
$$

where $A_{n+1, n}\left(B_{n+1, n}\right)$ means the hopping integral between the $n$th and $(n+$ 1)th sites and $A_{n, n}\left(B_{n, n}\right)$ the on-site energy at site $n$ in chain $A(B)$, and $V_{n}$ is the hopping integral from chain $A(B)$ to chain $B(A)$ at site $n$, respectively.

(a)

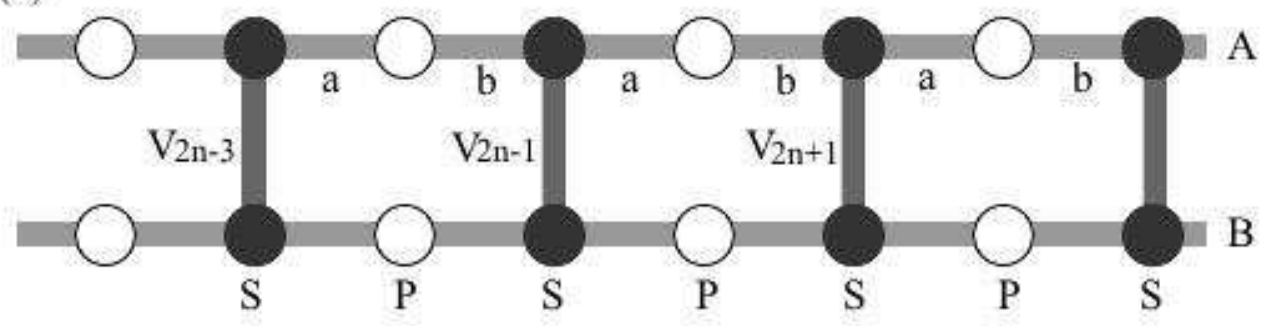

(b)

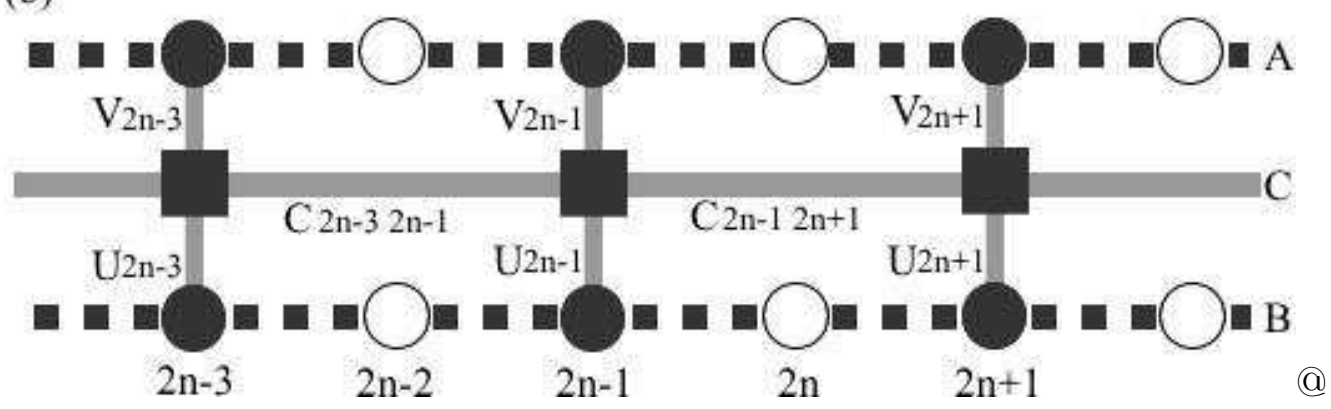

Fig. 1. Models of the double strand of DNA. (a) The two-chain model, (b) the three-chain model, where $S$ and $P$ represent sugar and phosphate sites, respectively.

Furthermore it can be rewritten in the matrix form,

$$
\left(\begin{array}{c}
\phi_{n+1}^{A} \\
\phi_{n+1}^{B} \\
\phi_{n}^{A} \\
\phi_{n}^{B}
\end{array}\right)=\left(\begin{array}{cccc}
\frac{E-A_{n n}}{A_{n+1 n}} & -\frac{V_{n}}{B_{n+1 n}} & -\frac{A_{n-1 n}}{A_{n+1 n}} & 0 \\
-\frac{V_{n}}{B_{n+1 n}} & \frac{E-B_{n n}}{B_{n+1 n}} & 0 & -\frac{B_{n-1 n}}{B_{n+1 n}} \\
1 & 0 & 0 & 0 \\
0 & 1 & 0 & 0
\end{array}\right)\left(\begin{array}{c}
\phi_{n}^{A} \\
\phi_{n}^{B} \\
\phi_{n-1}^{A} \\
\phi_{n-1}^{B}
\end{array}\right) \equiv T_{d=2}(n)\left(\begin{array}{c}
\phi_{n}^{A} \\
\phi_{n}^{B} \\
\phi_{n-1}^{A} \\
\phi_{n-1}^{B}
\end{array}\right)
$$

We would like to investigate the asymptotic behavior $(N \rightarrow \infty)$ of the products of the matrices $M_{d}(n)=\prod_{k}^{n} T_{d=2}(k)$ [37]. According to the parameter sets given by Iguchi [7], we set $A_{n+1, n}=B_{n+1, n}=a(=b)$ at odd (even) site $n$, 
respectively and $V_{n}=0$ at even sites (phosphate sites) for simplicity. The chain $A$ and $B$ are constructed by the repetition of the sugar-phosphate sites, and the inter-chain hopping $V_{n}$ at the sugar sites come from the nucleotide base-pairs, i.e., $A-T$ or $G-C$ pairs. (See Fig.1(a).)

The reduction to single-chain system $(d=1)$ and the extension to three-chain system $(d=3)$ are easy [38]. In particular, when we allow hop of the electron between the nearest neighbor nucleotide base-pairs by the overlap integral as well as hop between the backbone sites, the two-chain model can be easily extended to the three-chain one. The three-chaneels syatem can be described by following Schrödinger equation.

$$
\begin{aligned}
A_{n+1, n} \phi_{n+1}^{A}+A_{n, n-1} \phi_{n-1}^{A}+A_{n, n} \phi_{n}^{A}+V_{n} \phi_{n}^{C} & =E \phi_{n}^{A}, \\
C_{n+2, n} \phi_{n+2}^{C}+C_{n, n-2} \phi_{n-2}^{C}+C_{n, n} \phi_{n}^{C}+V_{n} \phi_{n}^{A}+U_{n} \phi_{n}^{B} & =E \phi_{n}^{C}, \\
B_{n+1, n} \phi_{n+1}^{B}+B_{n, n-1} \phi_{n-1}^{B}+B_{n, n} \phi_{n}^{B}+U_{n} \phi_{n}^{C} & =E \phi_{n}^{B},
\end{aligned}
$$

for the odd site $n$. On the other hands,

$$
\begin{aligned}
& A_{n+1, n} \phi_{n+1}^{A}+A_{n, n-1} \phi_{n-1}^{A}+A_{n, n} \phi_{n}^{A}=E \phi_{n}^{A}, \\
& B_{n+1, n} \phi_{n+1}^{B}+B_{n, n-1} \phi_{n-1}^{B}+B_{n, n} \phi_{n}^{B}=E \phi_{n}^{B},
\end{aligned}
$$

for the even sites $n$. The geometry and setting in the three-chain model is given in Fig.1(b).

\section{Correlated sequences}

As the correlated binary sequence $\left\{V_{n}\right\}$ of the hopping integrals, we use some real DNA sequence such as the bacteriophages of E.coli (phage- $\lambda$, phage-186), HCh-22 and histone H1. We can get the real DNA sequence from the gene data bases [39].

When we convert the nucleotide sequences $\left\{S_{n}\right\}$ to a numerical data $\left\{V_{n}\right\}$, some rules are used as seen in DNA walk analyses [27]: (I)Prine-pyrimidin rule. If $S_{n}$ is a purine (A or $\mathrm{G}$ ) then $V_{n}=W_{A G}$, if $S_{n}$ is a pyrimidin (C or T) then $V_{n}=W_{C T}$, where $W_{A G}$ and $W_{C T}$ denote moderate numerical values for calculation. (II)Hydrogen bond energy rule. $V_{n}=W_{G C}$ for strongly bonded pairs (G-C), $V_{n}=W_{A T}$ for weakly bonded pairs (A-T), where $W_{G C}$ and $W_{A T}$ denote moderate numerical values for calculation. (III)Hybrid rule. $V_{n}=W_{A C}$ for A or $\mathrm{C}, V_{n}=W_{G T}$ for $\mathrm{G}$ or $\mathrm{T}$, where $W_{A C}$ and $W_{G T}$ denote moderate numerical values for calculation. Apparently the hydrogen bond energy rule is

relevant in order to investigate the electronic localization in the sequence of 
DNA double helix. We apply the hydrogen bond energy rule to the real DNA sequences in the present paper.

Moreover, we compare the characteristics of the localization property with the result in an artificial sequence generated by following asymmetric modified Bernoulli map [40].

$$
X_{n+1}=\left(\begin{array}{cc}
X_{n}+2^{B_{0}-1} X_{n}^{B_{0}} & \left(X_{n} \in I_{0}\right) \\
X_{n}-2^{B_{1}-1}\left(1-X_{n}\right)^{B_{1}} & \left(X_{n} \in I_{1}\right),
\end{array}\right.
$$

where $I_{0}=[0,1 / 2), I_{1}=[1 / 2,1) . B_{0}$ and $B_{1}$ are the bifurcation parameters which control the correlation of the sequence, and we set $1<B_{0} \leq B_{1}<2$ for simplicity. The asymmetry of the map $\left(B_{0} \neq B_{1}\right)$ corresponds to the asymmetric property of the distribution of the real sequence of the double helix DNA that the number of the A-T pairs does not equal one of $\mathrm{G}-\mathrm{C}$ pairs which is different from the random binary sequence with equal weight. We introduce an indicator $R_{G C}$ for the rate of the G-C pair in the sequences as, $R_{G C}=\left(N_{G}+N_{C}\right) /\left(N_{G}+N_{C}+N_{A}+N_{T}\right)$, where $N_{G}, N_{C}, N_{A}$ and $N_{T}$ denote the number of each symbol G,C,A and $\mathrm{T}$ in the sequence, respectively.

In the ladder model we also use the symbolized sequences $\left\{V_{n}\right\}$ and/or $\left\{U_{n}\right\}$ by the following rule as the interchain hopping integral at odd sites $n$ :

$$
V_{n}=\left\{\begin{array}{l}
W_{A T}\left(X_{n} \in I_{0}\right) \\
W_{G C}\left(X_{n} \in I_{1}\right)
\end{array}\right.
$$

In the numerical calculation, $W_{G C}$ is set at a half of $W_{A T}$ for simplicity. $\left(W_{G C}=W_{A T} / 2\right.$.) Then the artificial binary sequence can be roughly regarded as the base-pair sequence as observed in the $\lambda$-DNA or the HCh-22. The correlation function $C_{o}(n)\left(\equiv<V_{n_{0}} V_{n_{0}+n}>\right)\left(n_{0}=1, n\right.$ is even ) decays by the inverse power-law depending on the value $B$ as $C_{o}(n) \sim n^{-\frac{2-B_{1}}{B_{1}-1}}$ for large $n\left(3 / 2<B_{i}<2\right)$. The power spectrum becomes $S(f) \sim f^{-\frac{2 B_{1}-3}{B_{1}-1}}$ for small $f$. We focus on the Gaussian and non-Gaussian stationary region $\left(1<B_{i}<2\right)$ that correspond to some real DNA base-pair sequence with $S(f) \sim f^{-\alpha}(0.2<\alpha<1)$. There are various ways to generate the correlated sequences as seen in study in one-dimensional disordered system with longrange correlation. We must pay attention to the statistical properties. For example, when we use the correlated random walk with Hurst index to generate the correlated sequence, the sequence must be rescaled by the variance of the fluctuation because the fluctuation diverges with the length of the sequence. However, in the stationary sequence generated by the modified Bernoulli map 
the fluctuation does not diverge because it takes only alternative values at the each sites $n$.

\section{Numerical Result}

We give the numerical result of the energy dependence of the Lyapunov exponents. The definition is given by,

$$
\gamma_{i}=\lim _{n \rightarrow \infty} \frac{1}{2 n} \log \sigma_{i}\left(M_{d}(n)^{\dagger} M_{d}(n)\right)
$$

where $\sigma_{i}(\ldots)$ denotes the $i$ th eigenvalue of the matrix $M_{d}(n)^{\dagger} M_{d}(n)[41]$. As the transfer matrix $T_{d}(n)$ is symplectic, the eigenvalues of the $M_{d}(n)^{\dagger} M_{d}(n)$ have the reciprocal symmetry around the unity as $e^{\gamma_{1}}, \ldots, e^{\gamma_{d}}, e^{-\gamma_{d}}, \ldots, e^{-\gamma_{1}}$, where $\gamma_{1} \geq \gamma_{2} \geq \ldots \gamma_{d} \geq 0$

Furthermore, it is found that for the thermodynamic limit $(n \rightarrow \infty)$ the largest channel-dependent localization length $\xi_{d}\left(=1 / \gamma_{d}\right)$ determines the exponential decay of the Landauer conductance of the system between metallic electrodes as $g(n)=\sum_{i}\left(\cosh \left(2 \gamma_{i} n\right)-1\right)^{-1} \rightarrow \exp \left(-2 \gamma_{d} n\right)$ in units of $2 e^{2} / h$ at zero temperature and serves as the localization length of the total system of the coupled chains [37]. Recently, the electron transport for the molecular wire between two metallic electrorodes has been also investigated by several techniques [42].

We consider the correlation effect on the localization property of the disordered case by using some real DNA sequences and the modified Bernoulli model. Then we used a sample with the system size $N=10^{5}$ for the numerical calculation in the modified Bernoulli map. Note that perfect periodicity exists in the deterministic (even) sites in our models. (See Fig.1.) We introduce another long-range correlation due to the base-pair sequence on the odd sites $V_{2 n-1}$.

It is generally known that the correlation of the sequence enhances the delocalization in the electronic states. In the asymmetric modified Bernoulli system characterized by the two-parameters, $B_{0}, B_{1}$, the correlation decay depends on $B_{1}$ in large sequences, because we set $B_{0} \leq B_{1}$. Figures 2(a) shows the energy dependence of the Lyapunov exponents $\left(\gamma_{1}\right.$ and $\left.\gamma_{2}\right)$ for some cases in asymmetric modified Bernoulli system. They are named as follows: case $(1) B_{0}=1.0, B_{1}=1.0$, case $(2) B_{0}=1.0, B_{1}=1.9$ and case (3) $B_{0}=1.7, B_{1}=1.9$. Apparently the case (1) is more localized than cases (2) and (3) in vicinity of the band center $|E|<1$. The comparison between the case (2) and case (3) shows the effect of the asymmetry of the map on 

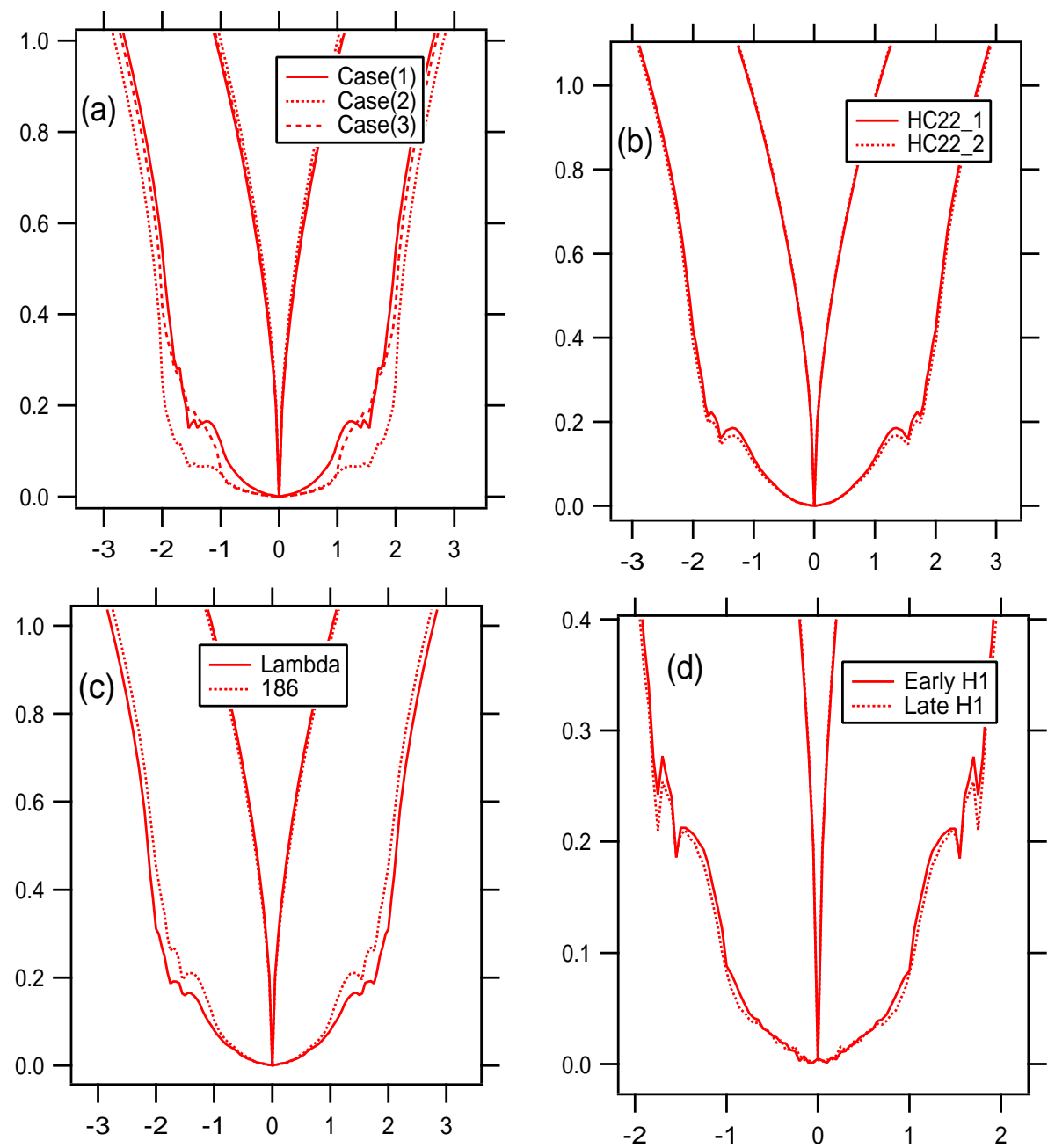

Fig. 2. Lyapunov exponents $\left(\gamma_{1}, \gamma_{2}\right)$ as a function of energy in the ladder model. (a) modeified Bernoulli model, (b) human chromosome 22, (c) bacteriophages of E.coli (phage- $\lambda$, phage-186), (d)early histone $\mathrm{H1}$ and late histone H1. The on-site energy is set at $A_{n n}=B_{n n}=0, a=-1.0, b=-0.5$. The size of the sequence is $N=10^{5}$ for (a), $N=10^{5}$ for (b), $N=48510$ for the phage- $\lambda$ in (c), $N=30624$ for the phage-186 in (c), $N=787$ for the early histone H1 in (d), and $N=1182$ for the late histone $\mathrm{H} 1$ in $(\mathrm{d})$.

the localization. $R_{G C} \sim 0.2$ for the case(2), $R_{G C} \sim 0.47$ for the case(3). In energy regime $|E|>1$, the Lyapunov exponent $\gamma_{2}$ in the case (2) is smaller than one in the case (3) in spite of the same correlation strength $B_{1}$. As a result, it is found that in the DNA ladder model the correlation and asymmetry enhance the localization length $\xi\left(\equiv \gamma_{2}^{-1}\right)$ of the electronic states around $|E|<1$, although the largest Lyapunov exponent $\gamma_{1}$ does not almost change by the effects.

Figure 2(b), 2(c) and 2(d) show the nonnegative Lyapunov exponents in real DNA sequences of (b)HCh-22, (c) bacteriophages of E. coli and (d)Histon pro- 
teins. In the case of HCh-22, we used two sequences with $N=10^{5}$, extracted from the original large DNA sequence. The result shows the Lyapunov exponents do not depend the details of the difference of the sequence in HCh-22. Although the weak long-range correlation has been observed in HCh-22 as mentioned in introduction, it does not affect on the localization property. The sequences we used are almost symmetric $\left(R_{G C} \sim 0.5\right)$.
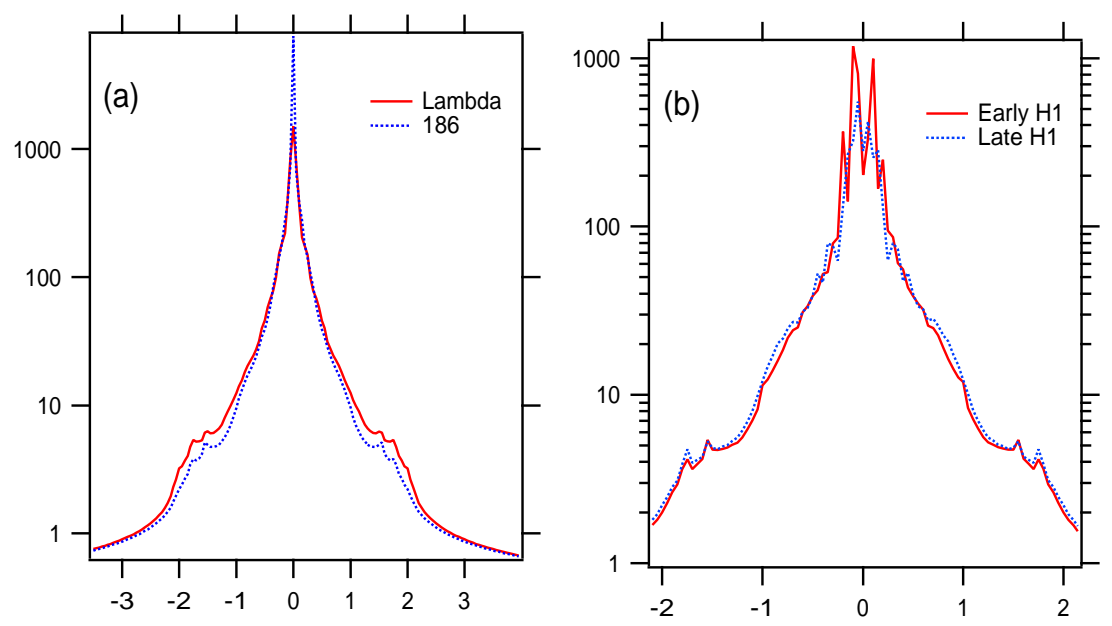

Fig. 3. Localization length $\xi\left(=\gamma_{2}\right)$ as a function of energy in the ladder model. (a) bacteriophages (phage- $\lambda$, phage-186), (b)early histone H1 and late histone H1. The parameters are same to those in Fig.2.

In Fig.2(c) and (d) the least nonnegative Lyapunov exponents are influenced by the difference in the sequence. The localization length $\xi_{d}=1 / \gamma_{d}$ defined by the least nonnegative Lyapunov exponent for the bacteriophages and histon $\mathrm{H} 1$ are shown in Fig.3(a) and (b). Apparently the localization length of the phage- $\lambda$ is larger than phage-186. Moreover, it seems that the difference between sequence of early histon $\mathrm{H} 1$ and late histon $\mathrm{H} 1$ effects on the resonance structure around $E=|2|$, although the difference does not change the localization property vicinity of the band center.

Furthermore, we have confirmed that almost similar property to the doublechain model have been observed in the three-chain model. Figure 4 shows the energy dependence of the Lyapunov exponents $\left(\gamma_{1}, \gamma_{2}, \gamma_{3}\right)$ in the three-chain model. In Fig.4(a) the result for asymmetric modified Bernoulli system is shown. Appearently, the correlation and/or asymmetry of the sequence effect a change in the second and third Lyapunov exponent. In contrast, although the global feature of $\gamma_{1}$ is almost unchanged, the local structure of the energy dependence is changed by the change of $B_{0}$. Figure $4(\mathrm{~b})$ shows the results in phage-fd and phage-186 in the three-chain model. It is found that the structure of the energy dependence around $|E|<2$ is different from that in the artificial sequence by the modified Bernoulli map. 

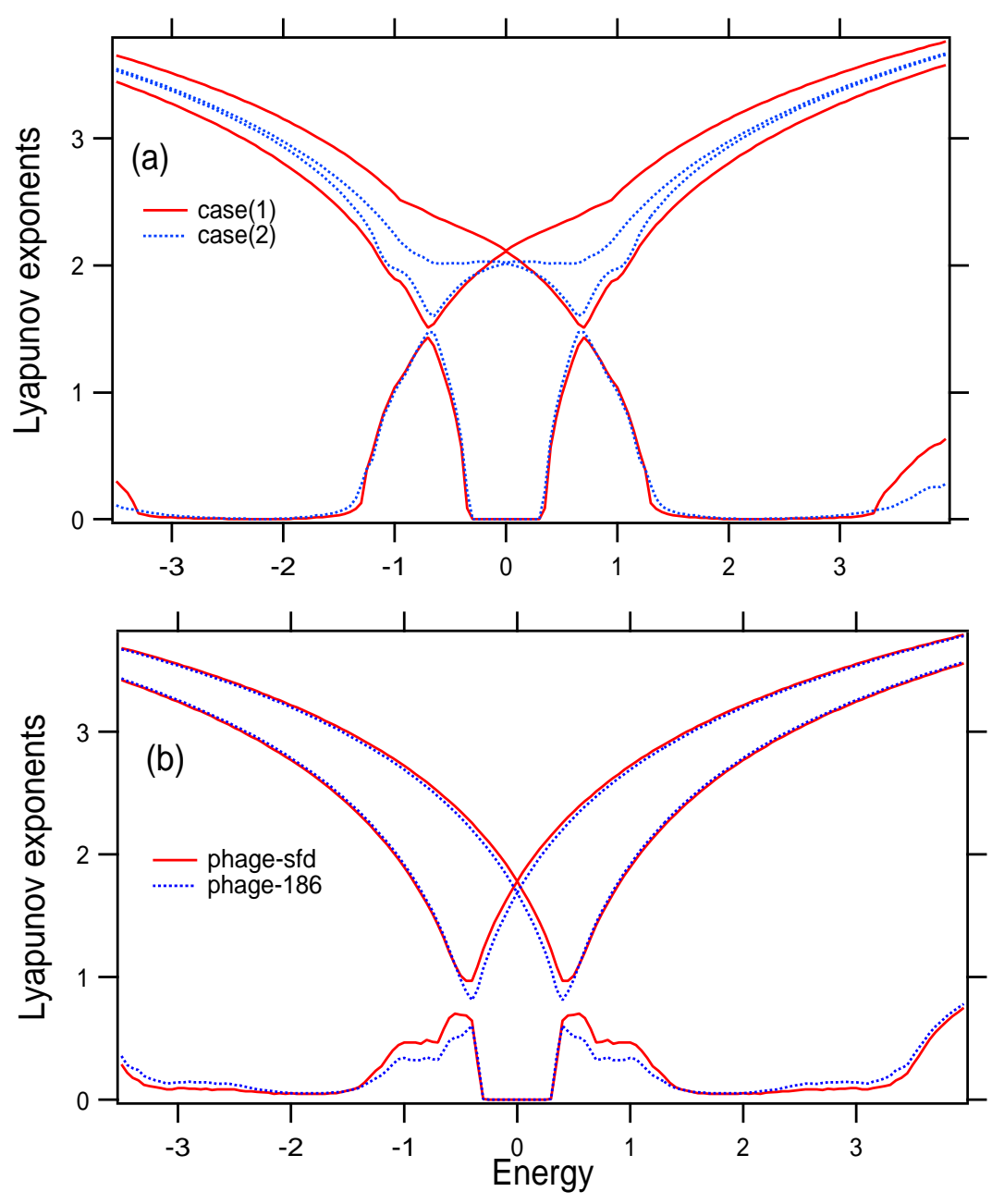

(a)

Fig. 4. Lyapunov exponents $\left(\gamma_{1}, \gamma_{2}, \gamma_{3}\right)$ as a function of energy in the three-chain model. (a) modeified Bernoulli model, (b)bacteriophages of E.coli (phage-fd, phage-186). The parameters are same as ones in Fig.2 except for on-site energies of C-chain $\left(C_{n, n}=0\right)$.

\section{Summary and Discussion}

We introduced two-chain and three-chain models as simple models for electronic property in the double strand of DNA. We numerically investigated the correlation effect on the localization property of the one-electronic states in the disordered two-chain (ladder) and three-chain models with the long-range structural correlation by means of asymmetric modified Bernoulli model and some real DNA sequences. As a result, the correlation enhances the localization length $\left(\gamma_{2}^{-1}\right)$ around $|E|<1$, although the $\gamma_{1}$ does not almost change. In addition to the correlation effect, the asymmetry of the sequence also enhances the localization length. Almost similar property to the two-chain model have been observed in the three-chain model. 
The relation between the correlation length of the DNA sequence and the evolutionary process is suggested $[31,43]$. Moreover, it is interesting if the localization property would be related to the evolutionary process. Up to now, although we consider the effect of the two points correlation of the sequence on the localization, the relation between the general complexity of the sequence and the localization property is also very interesting future problem $[44,45]$.

Finally, it should be noted that although in the present paper we have focused on the localization properties of the electronic states at zero temparature, with enhasis on the ladder geometry by the backbone and correlation of the DNA sequence, for the sake of simplicity, in the experiment of the conductance property of the DNA, both the temperature effect and the temperature dependence become important. Indeed, the finite temperature can also reduce the effective system size and leads to the changes in the transport property.

The author would like to thank Dr. Kazumoto Iguchi for stimulating and useful discussions.

\section{References}

[1] J.P. Lewis, T.E. Cheatham,III, E.B. Strikov, H. Wang and O.F. Sarkey, J. Phys. Chem. B107, 2581(2003).

[2] D. Porath, G. Curiberti and R. Difelice, Topics in Current Chemistry, 1 (2004).

[3] G. Paun, G. Rozenberg and A. Salomaa, DNA Computing -New Computing Paradigms, (Springer-Verlag, 1998)

[4] D. Porath, A.Bezryadin, S. de Varies and C. Dekker, Nature (London) 403, 635 (2000).

[5] P. Tran, B. Alavi, and G. Gruner, Phys. Rev. Lett. 85, 1564(2000).

[6] G. Cuniberti, L. Craco, D. Porath, and C. Dekker, Phys. Rev. B 65, 241314(R) (2002).

[7] K. Iguchi, Int. J. Mod. Phys. B11, 2405(1997): J. Phys. Soc. Jpn. 70, 593(2001).

[8] N. Kurita and K. Kobayashi, Comp. Chem. 24, 351(2000).

[9] R. Di Felice, A. Calzolari, E. Molinari and A. Garbesi, Phys. Rev. B 55, 045104(2001).

[10] Z. G. Yu and Xueyu Song, Phys. Rev. Lett. 86, 6018(2001).

[11] P. Carpena, P.B. Galvan, P.Ch. Ivanov and H.E. Stanley, Nature 418, 955(2002); ibid, 84, 764(2003). 
[12] S. Roche, Phys. Rev. Lett. 91, 108101(2003); S. Roche, D. Bicout, E. Macia and E. Kats, ibid, 91, 22810(2003).

[13] K. Iguchi, Int. J. Mod. Phys. B17, 2565(2003).

[14] K. Iguchi, Int. J. Mod. Phys. B, in press (2004), " $\pi$-electrons in a single strand of DNA: a phenomenological approach".

[15] D. Hennig, Euro. Phys. J. B 30, 211(2002): D. Hennig, J.F.R. Archilla and J. Agarwal, Physica D 180, 256(2003).

[16] J. Ladik, Phys. Rep. 313, 171(1999).

[17] L. Shen, Y.-J. Ye and J. Ladik, Solid State Commmun. 121, 35(2002).

[18] R. Bruinsma, G. Guner, M.R. D’Orsogna and J. Rudnick, Phys. Rev. Lett. 85, 4393(2000).

[19] E.I. Kats and V.V. Lebedev, JETP Letters 75, 37(2002).

[20] E.B. Starikov, J. Photochem. Photobiol. C3, 147(2002).

[21] P. Damle, A.W. Ghosh and S. Datta, Chem. Phys. 281, 171(2002).

[22] A. Troisi and G. Orlandi, J. Phys. Chem. B 106, 2093(2002).

[23] A. Rodriguez et al., Phys. Rev. Lett. 92, 27404(2003).

[24] Y.A. Berlin, A.L. Burin and M.A. Ratner, Chem. Phys. 275, 61(2002).

[25] D.N. LeBard, M. Lilichenko, D.V. Matyushov and Y.A. Berlin and M.A. Ratner, J. Phys. Chem. B 107, 14509(2003).

[26] R.F. Voss, Phys. Rev. Lett. 68, 3805(1992).

[27] S.V. Buldyrev, et al., Phys. Rev. E 51, 5084(1995).

[28] DNA and Protein Sequence Analysis: A Practical Approach, (IRL press, 1997), Ed. by M.J. Bishop and C.J. Rawlings.

[29] D. Holste, I. Grosse and H. Herzel, Phys. Rev. E64, 041917(2001).

[30] D. Holste, I. Grosse, S. Beirer, P. Schieg and H. Herzel, Phys. Rev. E67, 061913(2003).

[31] Y. Isohata and M. Hayashi, J. Phys. Soc. Jpn. 72, 735(2003).

[32] I. Grosse, P. Bernaola-Galvan, P. Carpena, R. RomJan-RoldJan, J. Oliver, H.E. Stanley, Phys. Rev. E65 041905(2002).

[33] H. Yamada, M. Goda and Y. Aizawa, J. Phys.:Condens. Matter 3, 10043(1991).

[34] F.A.B.F. de Moura and M.L. Lyra, Phys. Rev. Lett. 81, 3735(1998).

[35] H. Yamada, J. Phys. Soc. Jpn. Suppl. A 72, 123(2003): Phys. Rev. B 69, 014205(2004). 
[36] J. Ladik, Quantum Theory of Polymers as Solids, (Plenum Press, New York, 1988).

[37] A. Crisanti, G. Paladin, and A. Vulpiani, Products of Random Matrices in Statistical Physics (Springer-Verlag, Berlin, 1993), and references therein.

[38] H. Yamada, Int. J. Mod. Phys. B, in press(2004); cond-mat/0401351.

[39] See, for example, http://www.cf.ac.uk/biosi/staff/ehrmann/tools/dna/, http://www.genome.ad.jp/.

[40] Y. Aizawa, C. Murakami and T. Kohyama, Prog. Theor. Phys. Suppl. 79, 96(1984); Y. Aizawa, Chaos, Solitons and Fractals, 11, 263(2000).

[41] H. Yamada and T. Okabe, Phys. Rev. E63, 26203(2001).

[42] A. Tikhonov, R.D. Coalson and Y. Dahnovsky, J. Chem. Phys. 117, 567(2002).

[43] S. Ohno, Evolution by Gene Duplication, (Springer-Verlag, 1970).

[44] R. Badi and A. Politi, Complexity Hierarchical structures and scaling in physics, (Cambridge University Press 1997).

[45] H. Yamada, in preparation. 